\section{Coming out of the dark}

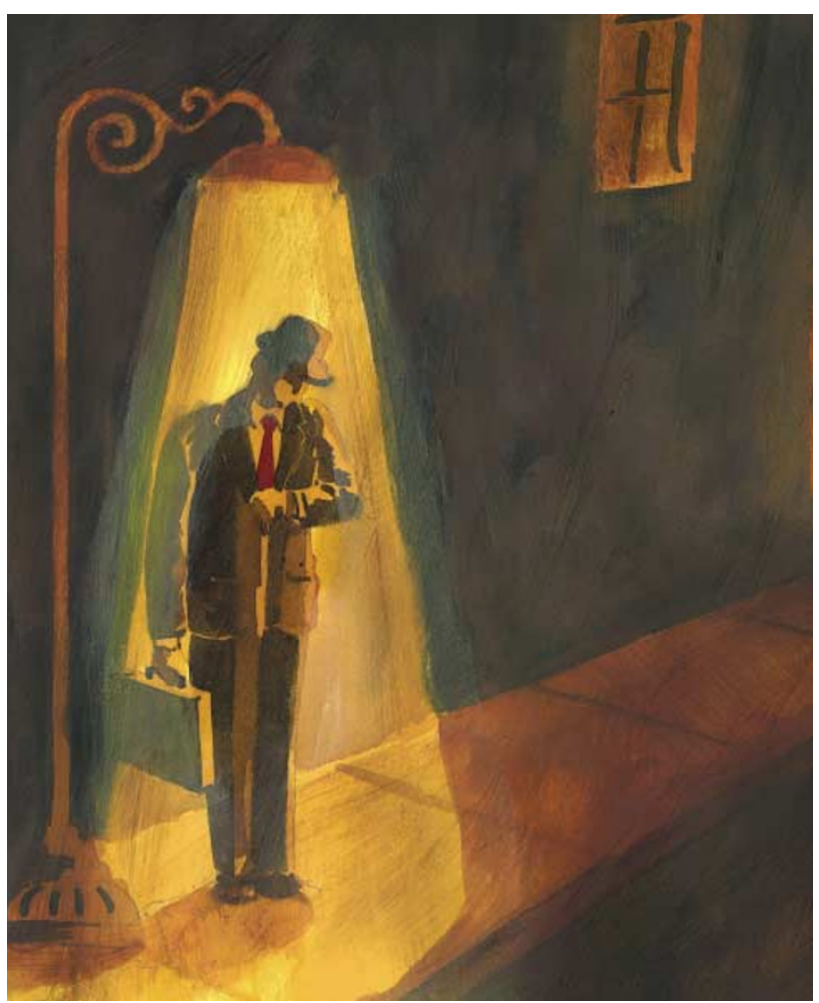

Seeds are tough cookies — they can withstand hostile surroundings until conditions favour germination. Emergent seedlings, though, are very vulnerable - particularly when first exposed to sunlight. Seedlings accumulate a small amount of protochlorophyllide, the precursor of chlorophyll, before they reach the surface so that, on sensing the first rays of sun, they are ready to photosynthesize. But too much protochlorophyllide can induce oxidative damage (in the form of bleaching), so chlorophyll biosynthesis must be carefully controlled. The identification, by Huq et al., of PHYTOCHROME-INTERACTING FACTOR-1 (PIF1), provides a mechanism by which this can be achieved.

Phytochrome (phy) receptors perceive light signals, which are thought to be transduced through PIFs to impinge on gene expression. The authors' investigations focused on PIF1, a basic helix-loop-helix (bHLH) transcription factor. Whereas pif1-insertion-mutant-seedlings showed no adverse effects when grown in the light from germination, those that were germinated and grown in the dark before being transferred to the light became bleached. This was reminiscent of a mutant phenotype that is caused by excess protochlorophyllide and, sure enough, protochlorophyllide levels were higher in the pif1 mutants that grew in the dark after germination. And the longer the time spent in darkness before transfer to light, the more severe the bleaching phenotype. This hinted that PIF1 might prevent the build-up of excess protochlorophyllide in the dark. Huq et al. indeed found that PIF1 negatively regulated the chlorophyll biosynthetic pathway - if pif1-mutant seedlings were grown in the dark for only a short period and then exposed to light before they had accumulated lethal levels of protochlorophyllide, they subsequently accumulated chlorophyll much faster than wildtype seedlings did in response to light.

The authors next established that PIF1 could bind to the so-called G-box DNA-sequence motif that is present in the promoters of many light-regulated genes. So what was the effect of PIF1 on transcription? It induced a marked increase in the activity of a luciferase reporter gene

\section{TECHNIQUE}

\section{A natural view}

Signalling proteins are under tight control both temporally and spatially to ensure that they carry out their roles correctly. For example, the small GTPase Cdc42 functions in processes such as cell motility, proliferation and apoptosis, so its activation at specific subcellular locations needs to be strictly regulated. It has been difficult to visualize Cdc42 activation dynamics in vivo, because of the limitations of present approaches. But, in Science, Hahn and colleagues now report the development of a biosensor that allows unlabelled, endogenous Cdc42 activation to be visualized in living cells.

They covalently labelled a domain from Wiskott-Aldrich syndrome protein (WASP) - a Cdc42 effector protein - with a dye that specifically reports protein interactions and protein conformational changes in living cells. The dye did not significantly perturb the Cdc42-WASP interaction, and it showed a threefold increase in fluorescence intensity on binding to Cdc42-GTP $\gamma \mathrm{S}$ (GTP $\gamma \mathrm{S}$ is a non-hydrolysable analogue of GTP). No increase was observed in the presence of Cdc42-GDP. The biosensor could also distinguish between $\mathrm{Cdc} 42$ and the related RhoA and Rac GTPases.

Although this biosensor could be used to measure Cdc42 activation in cell lysates, Hahn and co-workers developed a ratiometric imaging approach that allowed Cdc42 activation to be visualized in living cells. The final biosensor was named Mero-CBD - a merocyanine dye plus a Cdc42-binding domain.

The authors used this biosensor to monitor Cdc42 activation during cell adhesion and spreading. They found that $\mathrm{Cdc} 42$ is activated at the cell periphery, which extends filopodia, but not in actual filopodia. In addition, using specific inhibitors, they showed that Cdc42 activation at the cell periphery is microtubule dependent. They also found that $\mathrm{Cdc} 42$ is activated at the trans-Golgi apparatus: this indicates that it regulates the directional sorting/trafficking of polarity signals or that microtubules mediate the transport of activated Cdc42 to the cell periphery. Finally, they showed that increases and decreases in Cdc 42 activity are precisely coordinated spatially and temporally with cell extension and retraction.

So, Hahn and colleagues have developed a biosensor that gives us a natural view of Cdc42 dynamics - it allows the activation of endogenous protein to be detected at physiological concentrations in living cells, and does not require Cdc42 to be modified with a fluorescent label. This sensitive methodology could therefore be extended to "...proteins that cannot be derivatized or overexpressed for live cell studies", and could allow us to carry out a "...detailed kinetic analysis of rapid cellular processes".

(D) References and links Rachel Smallridge ORIGINAL RESEARCH PAPER Nalbant, P. et al. Activation of endogenous Cdc42 visualized in living cells. Science $\mathbf{3 0 5}$, 1615-1619 (2004)

FURTHER READING Erickson, J. W. \& Cerione, R. A Multiple roles for Cdc42 in cell regulation. Curr. Opin. Cell Biol. 13, 153-157 (2001)

\section{WEB SITE}

Klaus Hahn's laboratory: http://www.med.unc.edu/ wrkunits/2depts/pharm/faculty/hahn.htm 
when expressed in dark-incubated seedlings, and this transcriptional activity was suppressed in the presence of light treatment. PIF1 also interacted with the active (Pfr) form of phyA and phyB, the two main phytochromes that regulate the ability of seedlings to undergo 'greening' in response to light, and the lightinduced suppression of PIF1 activity required these phy proteins. As PIF1 can't interact with DNA and phyA or phyB concurrently, it seems that the phytoreceptors, when active, might function to modulate the activity of PIF1 — perhaps by sequestering or degrading it — so that chlorophyll biosynthesis can occur in the presence of light. So PIF1 seems to function as "a critical modulator by which plants optimize chlorophyll biosynthesis in response to environmental light conditions and protect against accumulation of potentially toxic levels of intermediates."

Katrin Bussell

\section{(D) References and links} ORIGINAL RESEARCH PAPER Huq, E. et al. PHYTOCHROME-INTERACTING FACTOR 1 is a critical bHLH regulator of chlorophyll biosynthesis. Science 305, 1937-1941 (2004)

FURTHER READING Quail, P. H. Phytochrome photosensory signalling networks. Nature Rev. Mol. Cell Biol. 3, 85-93 (2002)

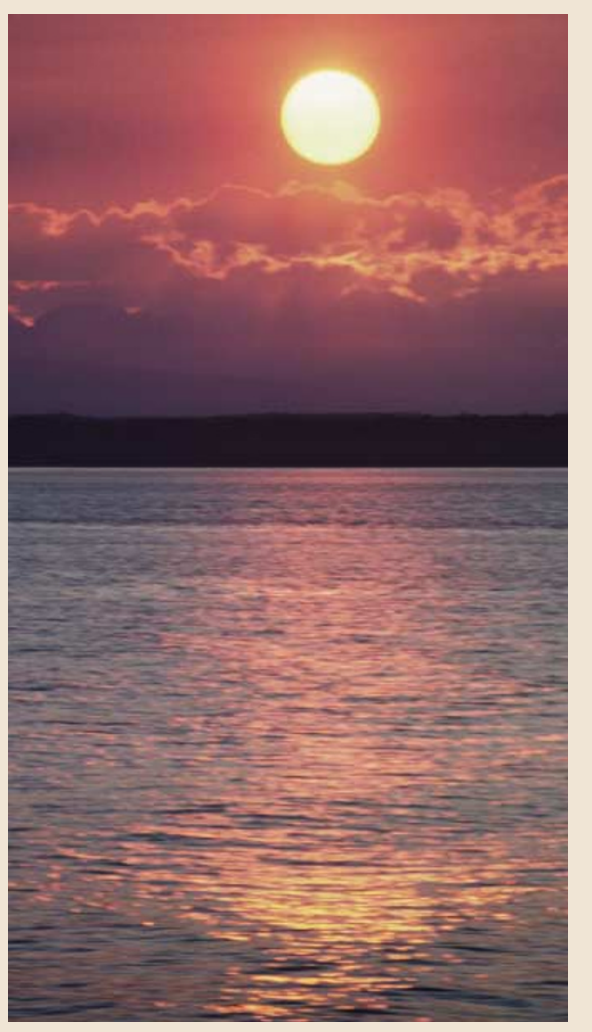

\section{Reverse control}

Post-translational modifications of histones, such as acetylation, phosphorylation and methylation, are thought to be dynamic and reversible. However, whereas the enzymes that help remove acetyl and phosphate groups from histone tails are known, those that counteract methylation have been elusive. But, two separate efforts have now led to the discovery that an enzyme, previously known to deiminate histone Arg residues, also regulates histone Arg methylation.

The enzyme, peptidylarginine deiminase- 4 (PADI4/PAD4), was known to catalyse the removal of an imino group from peptidyl Arg to produce peptidyl citrulline. In Science, Allis, Coonrod and coworkers now report that, when the substrate is methylated, PADI4/PAD4 demethyliminates the methylated Arg by releasing a methylamine group and generating citrulline.

Both groups examined the substrate specificity of PADI4/PAD4. In Cell, Kouzarides and colleagues report that, in vitro, PADI4/PAD4 deiminates Arg residues, specifically Arg2, 8, 17 and 26, in histone H3. Incidentally, Arg2, 17 and 26 are known substrates for the Arg methyltransferase CARM1. The Allis and Coonrod group showed, both in vitro and in vivo, that $\mathrm{PADI} 4 / \mathrm{PAD} 4$ targets include histone- $\mathrm{H} 3$ Arg8 and 17 and histone-H4 Arg3 (the latter is a known substrate for a different Arg methyltransferase known as PRMT1).

Using antibodies against specific methylated Arg residues, the Allis and Coonrod team showed that the PADI4/PAD4 activity does indeed 'undo' the methylation of Arg substrates. By tracing the radioactivity of the methyl group, they concluded that the methylimide group might be directly removed by demethylimination. Indeed, a dramatic decrease in the Arg methylation of histones was observed in response to PADI4/PAD4 activation in human HL-60 granulocytes. When treating granulocytes with PADI4/PAD4 small interfering (si)RNA, the level of histone-H4 Arg3 methylation remained the same and little citrulline was detected. This indicated to the Allis and Coonrod group that PADI4/PAD4 is the main enzyme responsible for regulating the levels of histone Arg methylation.

When they treated synthesized peptides with PADI4/PAD4, Kouzarides and co-workers noticed that dimethylated Arg peptides could not be converted to citrulline. Unfortunately, monomethyl Arg peptides were not available, but the decrease in methylation of histone $\mathrm{H} 3$, as measured by an antibody that is specific for monomethyl Arg, implies that only monomethylated Arg residues could be targeted by PADI4/PAD4.

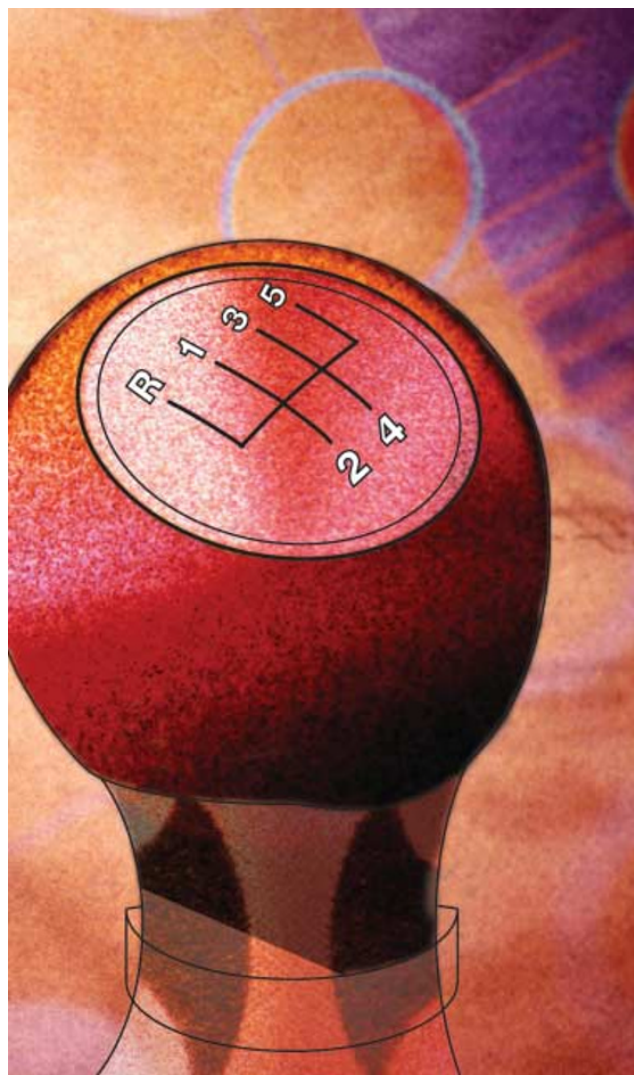

Arg methylation has been linked to transcriptional activation in response to hormone induction. Using an oestrogen-responsive promoter fused to a reporter gene, the Allis and Coonrod group showed that the presence of PADI4/PAD4 inhibited the hormone-stimulated reporter-gene activity, whereas a PADI4/PAD4 mutant protein failed to do so. As both groups confirmed, this transcriptional response coincides with the recruitment of PADI4/PAD4 to the promoter region, an increase in deiminated histone levels that is coupled to a decrease in methylated histone levels, and with the release of RNA polymerase II from the promoter.

To probe the mechanism by which PADI4/PAD4 antagonizes Arg methylation, the Kouzarides group tested a tail peptide from histone $\mathrm{H} 3$ in which Arg2, Arg8 and Arg17 were replaced by citrulline, and they found that the peptide remained unmethylated in the presence of CARM1. Together with the other findings, this indicates two possible mechanisms for PADI4/PAD4 action: first, it might deplete the histone-H3 substrate of CARM1, as implied by the Kouzarides group; or second, PADI4/PAD4 might reverse the methylation of monomethylated Arg. In addition, this raises the obvious question of how dimethylation of Arg residues is reversed...

Arianne Heinrichs

\section{(19) References and links}

ORIGINAL RESEARCH PAPERS Wang, Y. et al. Human PAD4

regulates histone arginine methylation levels via demethylimination. Science 2 Sept 2004 (doi:10.1126/science. 1101400) | Cuthbert, G. L. et al. Histone deimination antagonizes arginine methylation. Cell $\mathbf{1 1 8}$, 545-553 (2004) 